\title{
EVALUACIÓN DE PROFESORES UNIVERSITARIOS POR SUS LABORES DE INVESTIGACIÓN Y CREACIÓN
}

\section{Introducción}

Debido principalmente a las diversas áreas de actividad presentes en una universidad moderna, la evaluación global de académicos universitarios resulta muy compleja. Es difícil comparar un químico con un músico, un psicólogo con un arquitecto o un médico con un filósofo. Sin embargo, se pueden encontrar elementos comunes. A menudo una universidad necesita juzgar la calidad de sus profesores para cada área y, transversalmente, en las distintas áreas del saber que comprende. La evaluación de profesores es necesaria para la contratación, promoción, aumentos de salario, nombramiento en cargos especiales o premios. Es de vital importancia para una universidad establecer criterios adecuados de evaluación.

Los autores (académicos de las áreas de ingeniería, arquitectura, artes, astrofísica y un gestor académico) han tratado de encontrar criterios comunes y de advertir las diferencias para la evaluación de la investigación o creación en alguna de sus formas. Para ello se han nutrido de discusiones con académicos de otras áreas de artes, humanidades, ingenierías, ciencias básicas, ciencias naturales y ciencias sociales ${ }^{6}$.

Este artículo está organizado de la manera siguiente. La sección 2 presenta los objetivos; la 3 discute preguntas claves para la evaluación de la investigación y creación; las secciones 4 y 5 presentan los métodos de evaluación de ciencias y artes, respectivamente. En la sección 6 se exponen los criterios de evaluación por áreas disciplinarias y las conclusiones se presentan en la sección 7 .

6 Contexto de trabajo de la Comisión de Evaluación Académica UC 2009, que buscó establecer criterios para la evaluación de actividades académicas. 


\section{Objetivos}

El objetivo de este trabajo es definir un marco conceptual general para la calificación de profesores universitarios. Para ello, primero vamos a definir criterios generales para la evaluación académica de investigación y creación, transversales a todas las áreas del conocimiento, y luego definir y reconocer criterios específicos para la evaluación académica de investigación y creación en áreas disciplinarias representativas.

Este trabajo trata la evaluación de profesores universitarios por sus actividades de investigación o creación artística, en las cuales se genera conocimiento que se puede difundir, pero no incluye la evaluación de otras importantes actividades académicas como docencia, difusión o gestión académica. En particular, no se discute cómo evaluar la sinergia de la investigación con la formación de personas -íntimamente ligadas-, porque eso correspondería más al ámbito de la docencia.

\section{Preguntas clave para evaluar investigación y creación}

Preguntas clave respecto de conceptos básicos para evaluar en investigación y creación son, por ejemplo, qué se evalúa, quiénes deben evaluar y cómo se evalúa?

Hay cuatro etapas en el trabajo de investigación y creación universitaria:

- El proyecto o propuesta: etapa en la cual se formula el proyecto, pidiendo financiamiento o simplemente aprobación. Si la propuesta es exitosa, debe evaluarse positivamente al académico.

- El trabajo: etapa más importante, en la cual se desarrolla la generación de conocimiento (investigación o creación). Paradójicamente, esta etapa generalmente no se evalúa, ya que no existen criterios.

- La publicación o difusión de los resultados o productos: no siempre la investigación es exitosa o publicable, aunque es deseable que siempre tienda a difundir sus resultados. Esta etapa sí se evalúa 
y se consideran publicaciones, obras, patentes, interpretaciones, entre otros productos.

- El reconocimiento de pares: evauación a posteriori, a través de premios, nombramientos, por ejemplo. Esta etapa no se garantiza en todos los casos, pero si ocurre debe evaluarse y reconocerse como altamente positivo.

Quienes evalúan deben ser principalmente "pares representativos", con una trayectoria destacada en las actividades académicas que realizan, constituyendo buenos referentes. Asimismo, pueden considerarse también algunos referentes externos que se estimen relevantes (de áreas afines o de otros países). Esto importa sobre todo en áreas en las cuales la especificidad del conocimiento es mayor y, por tanto, también lo es la especialización, condición que muchas veces no se consigue en una comisión de evaluadores con pocos miembros.

Para decidir cómo evaluar se debe tener en cuenta la existencia de un balance entre cantidad y calidad del trabajo de cada académico. Del mismo modo, debiera existir un equilibrio entre objetividad y subjetividad, tanto del trabajo de los académicos de una determinada área como también de las distintas áreas disciplinarias, de manera que se puedan asegurar niveles de objetividad/subjetividad comparables entre ellas. Otro elemento que se constituye en un insumo de la evaluación es velar para que los procesos de calificación se realicen con criterios difundidos previamente (e idealmente sociabilizados). Además, se debe establecer un cierto grado de flexibilidad en la evaluación cuando ello signifique ir en beneficio del académico sin descuidar la equidad del proceso. Finalmente, también es importante la incorporación de parámetros éticos en la evaluación de investigación y creación. Para ello se pueden usar argumentos aceptados mundialmente (UNESCO, 2005; National Academy Press, 2004).

Adicionalmente se propone considerar una serie de puntos de orden general que pueden agregar valor al proceso de evaluación, entre otros: la vinculación con los planes de desarrollo del área dentro de la respectiva universidad, las diferencias intrínsecas con académicos 
de otras universidades del país y del mundo y la congruencia con los criterios de evaluación aplicados por las agencias que financian investigación científica o creación artística.

\section{Investigación en ciencias y su evaluación}

La investigación posee parámetros difundidos en la comunidad de investigadores, con elementos consensuados y de larga data para evaluar su actividad, hecho que contrasta, por ejemplo, con la creación en artes.

La actividad científica ha evolucionado a lo largo de los siglos ampliándose y diversificándose y creando nuevas áreas interdisciplinarias. Paradójicamente, la evaluación de la investigación científica últimamente está convergiendo: actores de diversas ciencias reconocen la importancia de adoptar criterios similares, aplicables transversalmente. En la ciencia actual hay parámetros de evaluación aceptados ampliamente, objetivos y cuantificables:

- Número de publicaciones: información que mide la producción científica, aunque hay distintos tipos de publicaciones: con arbitraje (ISI) -y por tanto con un filtro de calidad básico- y sin arbitraje (libros, capítulos de libros, contribuciones a congresos, resúmenes, cartas al editor, comunicaciones, entre otros).

- Número de citas (referencias bibliográficas): indicador que mide la aceptación entre los pares, o sea la calidad de la producción científica. Esto es difícil de aplicar en una evaluación de los últimos años, ya que las citas demoran en aparecer en la literatura.

- Índice de Hirsch (índice h): indicador que considera los dos anteriores, midiendo simultáneamente la cantidad y calidad de la producción científica sobre la base del número de publicaciones ISI y de sus citas (Hirsch, 2005; Ball, 2005; Ball, 2007). Este índice mide productividad del autor e importancia de su contribución científica; sin embargo, es más útil para medir la trayectoria científica a lo largo de toda la carrera que para evaluar la producción en un periodo breve.

- Número de proyectos científicos obtenidos competitivamente: indicador que mide también la cantidad de producción científica, aunque en algunos casos puede ser una medida del potencial 
más que de la trayectoria (pero que igual puede ser importante en algunas áreas o etapas de la carrera). Se puede medir, por una parte, el número de proyectos como investigador principal vs. como coinvestigador, y la cantidad de proyectos nacionales vs. internacionales, por otra, en ambos casos obtenidos competitivamente.

- Financiamiento total de los proyectos conseguidos competitivamente. Este índice podría interpretarse como una medida de la calidad de los proyectos.

Los dos primeros criterios son fácilmente medibles usando las bases de datos disponibles. En particular, algunas de estas bases pueden ser usadas para implementar fácilmente incentivos para las publicaciones que muchas universidades otorgan a sus profesores.

Una sugerencia para la evaluación en investigación sería cuantificar la producción científica contando las publicaciones más los proyectos. Esto es un criterio general que puede ser fácilmente implementado para las distintas áreas del conocimiento.

Para ello se puede privilegiar el número de publicaciones con arbitraje. Por ejemplo, que una publicación ISI valga cinco o diez veces más que una publicación sin peer review. Si se quiere ser más preciso, se puede contar el número de publicaciones del investigador como autor principal, aunque esto es más difícil de implementar porque el orden de los autores no refleja lo mismo en distintas disciplinas. Por ejemplo, en matemáticas se usa estricto orden alfabético; en biología el responsable del proyecto va al final y en física o astronomía se ubica primero el autor principal.

El número de proyectos de un investigador también puede ser evaluado, por ejemplo, dando el doble de importancia a los proyectos obtenidos como investigador principal que a los proyectos en los cuales figura como coinvestigador, o dando el doble o el triple de peso más a los internacionales que a los nacionales, considerando el tipo de proyecto adjudicado (tamaño y volumen de financiamiento). Estos criterios pueden ser ajustados para considerar los matices de las distintas universidades o áreas, aunque no es sano eliminarlos porque constituyen un buen control de calidad. 


\section{Creación en artes y su evaluación}

Desde siempre la manifestación artística ha sido percibida como un fenómeno personal y, por tanto, subjetivo y particular del creador. Por ello, el concepto de evaluación, que supone un juicio por parte del evaluador, ha sido implementado, aunque sostenidamente cuestionado, en los espacios donde se imparte la enseñanza artística.

Los criterios e instrumentos tradicionales de evaluación no siempre resultan asertivos y aplicables, ya que, aunque es factible adaptarlos, no logran cumplir cabalmente su cometido: una amplia gama de aspectos del proceso creativo en las actividades expresivas no queda reflejada en los resultados.

Para la creación en artes distinguimos artes visuales, escénicas, musicales y literarias, y artes cinematográficas y audiovisuales. Las artes visuales involucran dibujo, pintura, escultura, artes gráficas, fotografía, artes del fuego, arte digital, net-art, instalaciones, diseño (sin desentender su dimensión funcional) y, en alguna medida, la arquitectura (en lo que a su componente proyectiva se refiere). Las artes escénicas incluyen teatro, danza y performance. Las artes musicales comprenden dirección, composición, interpretación, canto coral, ópera y nuevas tecnologías. Las artes literarias narrativa, poesía, ensayo, cuento y novela. Las artes cinematográficas y audiovisuales abarcan el cine, en todos sus géneros, y el video.

En términos generales, la creación y sus procedimientos no son ajenos a los diversos ámbitos del trabajo humano. Allí donde se ha creado una idea o un producto físico nuevo, se han empleado cruces de ideas preexistentes (teorías), materiales o formas de trabajo que, bajo una nueva mirada o un nuevo uso, dan por resultado nuevas aplicaciones, ideas o formas. La creatividad es pues el resultado de una modalidad de pensamiento no necesariamente lineal, propia de un pensamiento divergente y cuestionador (Goleman, Kaufman y Ray, 2000). La ciencia y las artes encuentran en este punto una vinculación y semejanza, y sólo es posible entenderlas por separado cuando las metodologías de trabajo y la posible sistematicidad de los procedimientos que operan para la obtención de los resultados los convierten en ciencia (ciencias sociales y ciencias básicas) o arte. 
Se entiende por creación, en el ámbito de las artes y en cualquier disciplina, a un resultado concreto inédito -señalado en este caso como obra- que tiene por centro la metáfora, la analogía, la sensibilidad o la razón, y que, basado en procesos de elaboración no necesariamente repetibles, estructura un cuerpo de obra con códigos no siempre lineales. En estos resultados caben obviamente los procesos de elaboración de obras en diseño, arquitectura, artes visuales, teatro, literatura, danza, cine y música, todos los cuales tienen su particular método, material, forma y resultado diverso. De esta manera, los procesos creativos, si bien poseen rasgos comunes, poseen también numerosas diferencias dadas por la disciplina, las personas y los ámbitos (culturales, sociales, ideológicos) en los cuales se desarrollan.

La creación de una obra implica transfigurar, recomponer, reordenar un material y una experiencia, recurriendo a la repetición o variación de procedimientos técnicos e instrumentales de diversa índole (técnicas), dando como resultado un producto que varía, en relación con otros preexistentes, en sus temas, formas, imágenes, sonidos, textos, estructuras o problemas, otorgándose a sí mismo nuevas reglas, nuevos órdenes y estándares, ampliando de esta manera el horizonte de trabajo metafórico que ha trazado un conjunto de la sociedad. No puede quedar de lado en este proceso el contenido ético y valórico de sus procesos y resultados, lo que establece uno de los principales rasgos distintivos de hacer arte en una universidad.

Si bien las disciplinas universitarias vinculadas con las ciencias han establecido procesos de evaluación de la producción investigativa, lo han hecho en función de indicadores acordados en una comunidad científica amplia que posee parámetros internacionales estandarizados. No obstante la existencia de una comunidad de pares igualmente amplia, un proceso como ese es impensable -o al menos impracticable- en el mundo de las artes. Los parámetros cualitativos para evaluar la producción en el área creativa son tan múltiples como disímiles y varían con mucha sensibilidad, dependiendo de los desplazamientos sociales, geográficos e ideológicos en los cuales ubiquemos dicho proceso creativo y su correlativa evaluación (Souriau, 1990). Justamente, la riqueza de formas en que se manifiesta 
la cultura artística hace impracticable la estandarización de dichos procesos. Someterla a un canon atentaría contra ese rasgo principal del proceso de creación artística: su carácter inédito trata de romper, ciertamente, los moldes que se le tratan de imponer. Siguiendo a Kant, podemos decir que la facultad de juzgar en medio del placer es lo que denominamos "gusto", y ese procedimiento nos indica que estamos ante un juicio estético (Kant, 2007), por lo cual resulta imposible construir una norma que unifique la calidad.

Discriminar entonces entre la presencia o ausencia de buena calidad resulta -en el terreno de las artes- un problema que sólo es posible resolver -en parte- si en una universidad se acordara la paradoja de establecer una norma respecto a lo que los artistas deben crear, lo que de suyo atenta contra la práctica misma del arte.

Buena parte de la creación de obra encuentra su validación en la sola ejecución, al margen de elementos validadores externos que, tal como ya se dijo, siempre poseen un rasgo subjetivo, ya sea por condición social, política (ideológica), estética, técnica o geográfica. La calidad de una propuesta de obra dependerá siempre de algún rasgo, propiedad o característica que la valide en un contexto específico. Esto configura un entorno epistemológico complejo a la hora de establecer indicadores fiables, válidos y únicos.

De este modo, es posible establecer indicadores concretos que midan la calidad e impacto de un texto dramatúrgico o la interpretación de una obra musical; pero no encontramos tal precisión a la hora de evaluar una obra en fotografía, una obra en video o una escultura. Asimismo, un recurso validador indirecto puede ser la consideración valórica del lugar de emplazamiento o manifestación de la obra.

Por esa causa, la evaluación del desempeño de los artistas en una universidad exige algunas medidas e indicadores variables y -por qué no- instrumentos y formas de evaluación de diverso tipo, según el tipo de obra que se trate. Será importante además evaluar al académico en función de su capacidad de ser activo en su disciplina artística, comprobable mediante datos de su producción. Aunque parezca obvio, esto es necesario explicitarlo con claridad para evitar 
confusión en el proceso de calificación académica: se evalúa al académico artista por medio de su producción de obra, no la obra desvinculada de su autor.

Un proceso de evaluación de la producción de obra de un artista, académico de una universidad, idealmente debiera considerar la representatividad de expertos sobre la amplia gama del trabajo evaluado. Esto posibilita respetar las diferencias de la práctica artística, organizando grupos de evaluadores que, como pares, manejen profundamente los sistemas, procesos, espacios, medios, tecnologías, temas, recursos humanos o procedimientos, como factores de visibilidad o materialización de la producción artística.

\section{Criterios de evaluación por áreas disciplinarias}

Hemos elaborado un esquema que permite comparar criterios de evaluación de la investigación para las distintas disciplinas y que lleva a reconocer similitudes entre algunas de ellas. Este esquema, presentado en la tabla 1 , si es debidamente consensuado con académicos representantes de cada disciplina, permitiría comparar los criterios de evaluación de la investigación y creación.

La clasificación de áreas utilizada está basada en gran parte en la confeccionada por el Ranking Mundial de Universidades (Times Higher Education Supplement-QS), que establece la siguiente agrupación de áreas del conocimiento: Ciencias Sociales, Artes y Humanidades, Ciencias Naturales, Ciencias de la Vida y Biomédicas, Tecnologías e Ingenierías. Existen otras clasificaciones como la del Academic Ranking of World Universities del Institute of Higher Education, Shanghai Jiao Tong University, que agrupa las áreas del conocimiento en: Ciencias Sociales, Ingenierías/Tecnologías, Ciencias Naturales y Matemáticas, Medicina y Farmacia, y Ciencias de la vida y Agronómicas.

Utilizando los distintos criterios, cada institución puede completar el cuadro en la tabla 1 de acuerdo con sus áreas específicas. Se puede simplemente marcar con una " $x$ " el criterio que se debe aplicar para determinada área o asignar prioridades. Sugerimos que asignar prioridades es más definido y riguroso. Un departamento de 
Física podría decidir, por ejemplo, que publicar en una revista con arbitraje es muy importante, publicar un capítulo de libro es poco importante y publicar un artículo en el diario es nada importante.

El esquema de la tabla 1 cumple además la función de beneficiar al académico que puede planear sus actividades de investigación y creación. Sabiendo un profesor de matemáticas que se contarán sus publicaciones arbitradas y citas, no destinará tanto tiempo a otras actividades tales como capítulos de libros. Si no sabe de antemano qué es lo más importante para su evaluación, a menudo un profesor que está siendo evaluado encuentra subjetivas o arbitrarias las decisiones tomadas, o dedica tiempo en actividades de menor relevancia para la institución.

Cabe destacar que de la profundización en cada disciplina pueden surgir otros nuevos criterios para evaluar. Además, esta tabla no incluye formación de personas, porque eso correspondería más al ámbito de la docencia. Se podría agregar como criterio el número de tesis dirigidas, si se quiere considerar, por ejemplo, la sinergia con las actividades docentes.

Al completar el cuadro de la tabla 1 sugerimos, primero, no hacer demasiado exigentes los requerimientos de un área que la puedan perjudicar en la competencia por recursos contra las otras áreas. Una institución no quiere tener grandes diferencias de nivel entre las distintas áreas. Segundo, no hacer demasiado "livianas" las exigencias de un área, atentando contra la calidad y perjudicando eventualmente su desarrollo futuro. Esto aseguraría que el proceso de calificación se oriente a retroalimentar mejoras crecientes del nivel de investigación y creación.

La tabla 1 contiene criterios generales comunes a ciencias y artes, como resulta ser la difusión o publicación en circuitos reconocidos, el reconocimiento de pares, el levantamiento de fondos y las redes de colaboración y su impacto. 
242 EVAlUACIÓN DE PROFESORES UNIVERSITARIOS - Dante Minniti, Pablo Irarrázaval, Ignacio Villegas, Fernando Pérez, Mauricio Ferrari

Tabla 1: Criterios de investigación y creación para distintas áreas

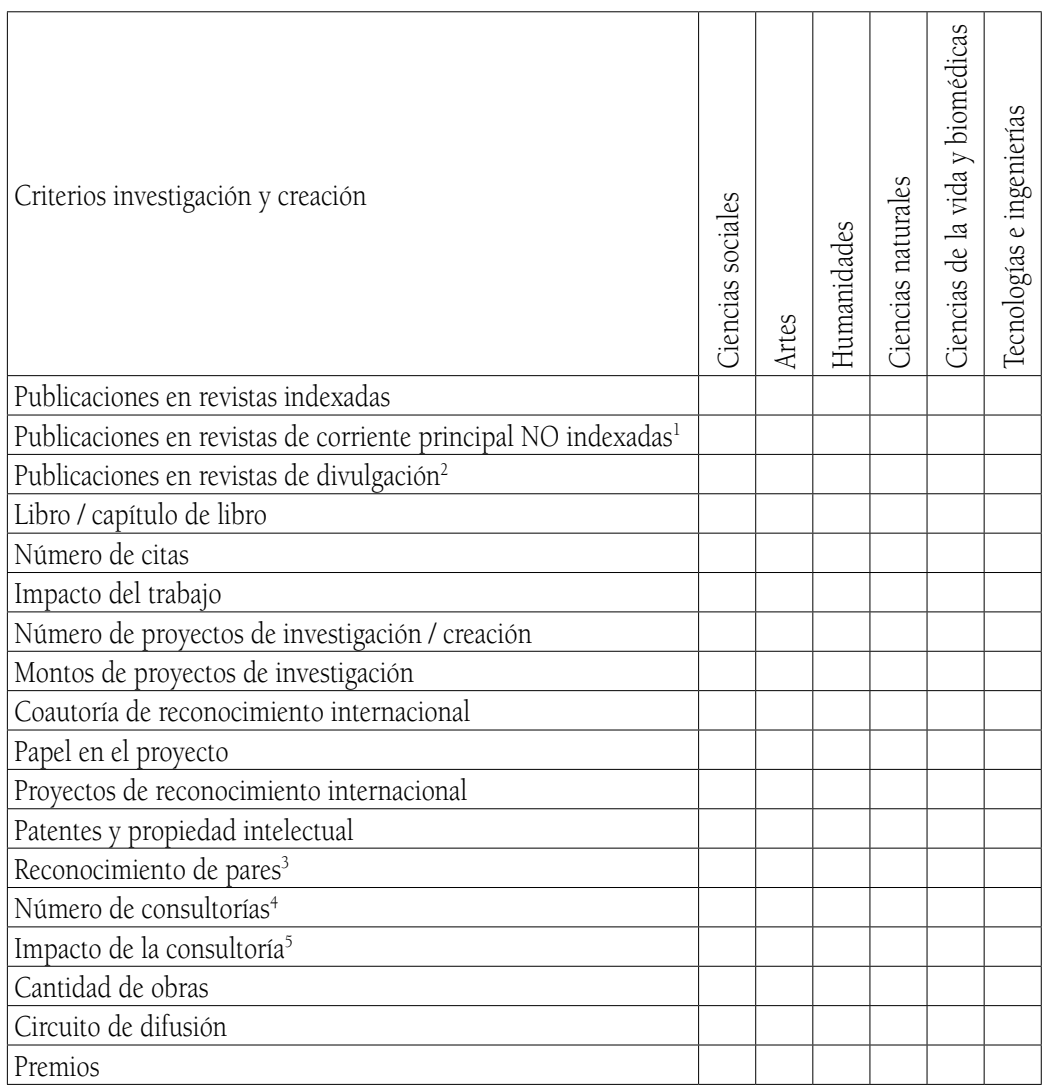

Notas de Tabla 1:

1 Referidas a revistas que poseen importancia en la disciplina y que por alguna razón justificada no tienen indexación.

2 Las revistas de divulgación corresponden a publicaciones que contienen un conjunto de artículos e imágenes sobre distintos temas de interés general y eventos de actualidad. Generalmente aparecen semanal, quincenal o mensualmente, y pueden presentarse en formato impreso o electrónico.

3 El reconocimiento de pares se puede dar de muchas formas. Se pretende que sea recogido mediante reconocimientos explícitos y objetivos, como por ejemplo la invitación a ser jurado en concursos para fondos de investigación y/o creación, o bien la participación en comités editoriales de revistas de relevancia.

4 Consultorías que generan conocimiento y que son reconocidas por los pares.

5 Impacto objetivo y medible, que genera conocimiento producto del desarrollo profesional de una determinada actividad. 


\section{Conclusiones}

En este trabajo se describen y contrastan las evaluaciones en las ciencias y en las artes para académicos universitarios. Se presentan criterios generales para la evaluación académica de investigación y creación, muchos de los cuales resultan ser transversales a todas las áreas del conocimiento. Entre ellos se encuentran la difusión en circuitos reconocidos, el reconocimiento de pares, el levantamiento de fondos, las redes de colaboración y su impacto. Se han reconocido además criterios específicos en un esquema para la evaluación académica de investigación y creación, propios de las áreas del saber universitario.

Este trabajo pretende ser un marco conceptual general y de base para las comisiones de calificación académica de una universidad, referidas a la actividad de evaluación en investigación y creación. Dichos criterios se pueden adaptar a la realidad de cada área de conocimiento y al entorno específico de cada universidad.

\section{Referencias bibliográficas}

Ball, P. (2005) Index aims for fair ranking of scientists. Nature, 436, p. 900.

Ball, P. (2007) Achievement index climbs the ranks. Nature, 448, p. 737.

Goleman, D.; Kaufman P. y Ray, M. (2000) El espiritu creativo. Buenos Aires: Ediciones B.

Hirsch, J. E. (2005) An index to quantify an individual's scientific research output. Proc. Nat. Acad. Sci., 46, pp. 165-169.

Kant, I. (2007) Crítica del juicio. Madrid: Espasa-Calpe.

On being a scientist: responsible conduct in research. (1995) National Academy Press, second edition. Available at http://www.nap.edu/catalog/4917.html

Souriau, E. (1990) Vocabulario de estética. Paris: P.U.F.

UNESCO (2005) Universal declaration on bioethics and human rights. Division of ethics of science and technology, UNESCO.

Recibido: 31 de agosto de 2009

Aceptado: 8 de octubre de 2009 